\title{
Does the Donor-Acceptor Concept Work for Designing Synthetic Metals? 1. Theoretical Investigation of Poly(3-cyano-3'-hydroxybithiophene)
}

\author{
Ulrike Salzner $^{\dagger}$ \\ Department of Chemistry, Bilkent University, 06533 Bilkent, Ankara, Turkey \\ Received: January 16, 2002; In Final Form: March 30, 2002
}

\begin{abstract}
Homo- and copolymers of hydroxythiophene and cyanothiophene have been investigated by employing density functional theory with the aim of determining the effect of donor-acceptor substitution on the electronic structure. The band gap of the copolymer is $0.11 \mathrm{eV}$ smaller than that of polythiophene. Bandwidths of valence and conduction bands are reduced by 0.22 and $0.36 \mathrm{eV}$ compared to polybithiophene. Conductivity after $\mathrm{p}$ - and n-doping could therefore be less than that of polythiophene. All properties of the copolymer are averages between those of the homopolymers. The charge separation between hydroxy- and cyano-substituted rings is $0.12 \mathrm{e}$ in the neutral state and $0.13 \mathrm{e}$ and the dication. The ionization potential and electron affinity of poly(hydroxythiophene) are 1.78 and $1.63 \mathrm{eV}$ smaller than those of poly(cyanothiophene). According to the donor-acceptor concept, a decrease in band gap and an increase in bandwidths compared to the homopolymers should have resulted. We rationalize the absence of band broadening with reduced interaction between fragments with very different energies in agreement with perturbation theory.
\end{abstract}

\section{Introduction}

The donor-acceptor concept for band gap engineering in conducting polymers was proposed by Havinga et al. in $1993 .{ }^{1}$ The basic idea is that a regular alternation of conjugated donor and acceptor moieties in a conjugated chain leads to broadening of valence and conduction bands and induces small band gaps. Band gap decrease and bandwidth increase were predicted to become stronger with increasing electronegativity difference between donor and acceptor. Havinga et al. tested their hypothesis by employing squaric acid and croconic acid as acceptors and heteroring systems with nitrogen and sulfur atoms as donors. Band gaps down to $0.5 \mathrm{eV}$ and intrinsic conductivities of up to $10^{-5} \mathrm{~S} / \mathrm{cm}$ were obtained. The highest conductivity upon doping was $1 \mathrm{~S} / \mathrm{cm}$.

Since small band gaps are the necessary condition for intrinsic conductivity and wide bandwidths are needed for high on-chain mobility, Havinga's proposal led to increased interest in donoracceptor systems, ${ }^{2-41}$ although several investigations on copolymers had been published prior to the 1993 paper. ${ }^{42-53}$ The method has been employed successfully for band gap adjustment between the values of the homopolymers, ${ }^{5,19,42,48,50}$ for tuning the color of light emitting systems,${ }^{29,34,35,39,47}$ for increasing the efficiency of photoluminescence, ${ }^{35,44,47}$ for creating quantum well structures, ${ }^{5,19,44,46,47}$ and for increasing intrinsic conductivity by reducing band gaps compared to those of the corresponding homopolymers. ${ }^{7,8,26,36}$ Although intrinsic conductivities of up to $10^{-3} \mathrm{~S} / \mathrm{cm}^{26}$ are impressive, the highest conductivity upon doping ${ }^{1,7-9,13,15,26,36,49,50}$ reported for a donor-acceptor system is, to our knowledge, $4.8 \mathrm{~S} / \mathrm{cm}^{8}{ }^{8}$ Homopolymers seem to conduct better, e.g., polyacetylene (PA) $10^{5} \mathrm{~S} / \mathrm{cm},{ }^{54}$ poly ( $p$-phenylene)

$\dagger$ Tel: (312) 290-2122. Fax: (312) 266-4579. E-mail: salzner@ fen.bilkent.edu.tr.
(PPP) $15 \times 10^{3} \mathrm{~S} / \mathrm{cm},{ }^{55}$ poly(3-methylthiophene) $(\mathrm{PMT}) 2000$ $\mathrm{S} / \mathrm{cm},{ }^{56}$ and polypyrrole (PPy) $1000 \mathrm{~S} / \mathrm{cm}^{55}$ Doping of homopolymers can yield an increase in conductivity of 15 orders of magnitude. In donor-acceptor systems conductivity increases by merely $2-5$ orders of magnitude. . $^{26,36}$

Copolymers of heterocycles with different ionization potentials (IPs) and electronegativities (EAs) have been studied theoretically prior to the Havinga paper. In most cases properties (including band gaps) are intermediate between those of the homopolymers and band gaps can be tuned between those of the homopolymers by changing composition. ${ }^{10,43,48,53}$ Poly(aminosquaraine), a model for the compounds synthesized by Havinga et al., was analyzed theoretically by Tol and Brocks. 2,6,11 The authors concluded that donor-acceptor systems have small band gaps only if there is weak interaction between the two units. In the case of strong interaction, charge transfer increases the band gap. Weak interactions, however, lead to narrow rather than wide bands. Poly(aminosquaraine) was shown to be a special case in which symmetry prevents interaction between the nitrogen $\pi$-orbital and the squaraine fragment in the valence band. This leads to a reduced charge shift and allows for a small band gap and significant dispersion at the same time. In the absence of such special effects, donor-acceptor substitution was shown to lead to charge shift and $\pi$-electron confinement. ${ }^{17,48}$ Electron confinement is reflected in small bandwidths of valence and/or conduction bands. Narrow bands have been found theoretically in several donor-acceptor systems. ${ }^{2,38,57}$ Small dispersion leads to a smaller decrease of band gaps upon an increase in chain lengths. This has been observed experimentally. ${ }^{31}$ Small bandwidths can account for the lower conductivities of donor-acceptor systems upon doping compared to homopolymers and for low n-type conductivity that was attributed to dedoping in experimental work. 
It seems that the original prediction that donor-acceptor substitution leads to small band gaps and wide bandwidths at the same time does not hold in general. This is an important issue, since design of good conductors requires high on-chain mobility and therefore wide bands. If one aims to design systems with high conductivity, it is of little use to decrease band gaps at the expense of bandwidths, since this would decrease carrier mobility, which is already the major limitation of conducting polymers. In contrast, donor-acceptor substitution proved very useful for improving quantum efficencies for electroluminescence where electron confinement is desirable. To investigate the effect of donor-acceptor substitution on band gaps, bandwidths and conductivity, we started systematic investigations on various types of donor-acceptor systems. In this paper we present analysis of poly(3-cyano-3'-hydroxybithiophene). The copolymer is compared to polythiophene as well as to the corresponding homopolymers. A very similar polymer, poly(3-cyano-3'-methoxybithiophene) was studied experimentally. Ionization potential and band gap were shown to be about the same as in polythiophene but charge localization was found during p-doping. ${ }^{5,19}$ Investigation of a small-band gap donoracceptor polymer with high intrinsic conductivity is reported in the paper following this one. Work and systems with aromatic donors and quinoid acceptors is in progress.

\section{Methods}

Cyano- and hydroxythiophene were optimized and their energy levels were compared to those of thiophene. Monomers through tetramers of bithiophene (1), 3,3'-dicyanobithiophene (2), 3,3'-dihydroxybithiophene (3), and 3-cyano-3'-hydroxybithiophene (4) were optimized in planar geometry. For all

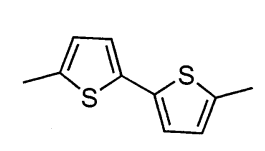

1

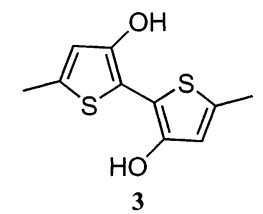

3
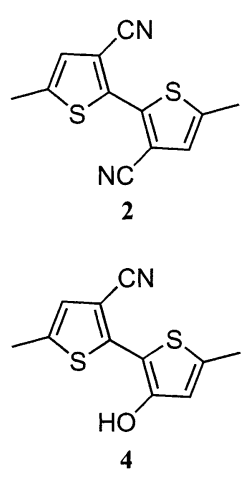

calculations density functional theory (DFT) was used. For the exchange-correlation functional Becke's three-parameter hybrid functional ${ }^{58}$ was combined with Perdew and Wang's correlation functional. ${ }^{59}$ The weight of the Hartree-Fock exchange was increased to $30 \%$ since such a functional yields HOMO-LUMO gaps in close agreement with $\lambda_{\max }$ values from UV spectroscopy. ${ }^{60}$ Stevens - Basch-Krauss pseudopotentials ${ }^{61}$ and split valence plus polarization basis sets were employed. ${ }^{61}$ At this level of theory, IPs and EAs are about $1 \mathrm{eV}$ too low for oligomeric $\pi$-systems but trends are reproduced correctly. The use of DFT orbital energies is justified from a pragmatic point of view although the exact physical meaning of DFT orbital energies is a controversial subject ${ }^{62-74}$ that is beyond the scope of the present investigation. Polymer properties were evaluated by plotting data for oligomers with increasing chain length against $1 / n$, the number of repeat units, and extrapolation by second-degree polynomial fitting. Electronic structures were analyzed with the natural bond orbital (NBO) method. ${ }^{75-79}$ All calculations were performed with Gaussian 98 Windows and

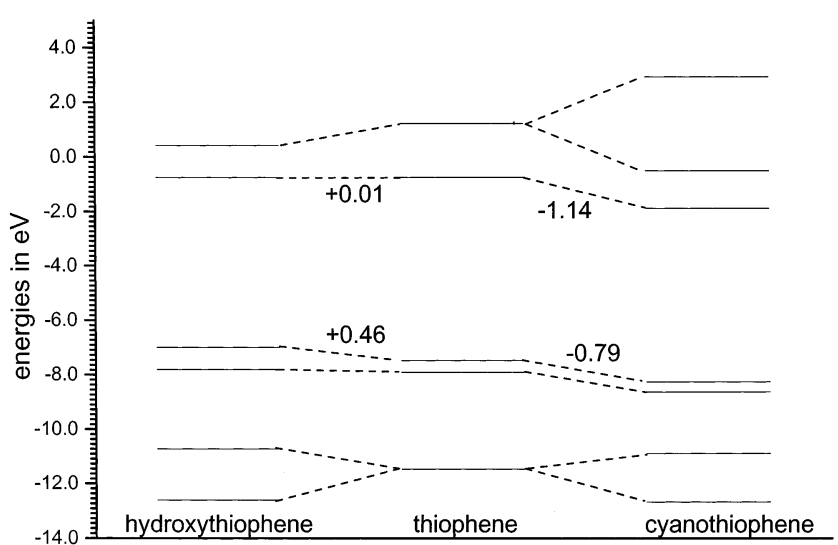

Figure 1. Influence of hydroxy and cyano substitution on the energy levels of the $\pi$-orbitals of thiophene.

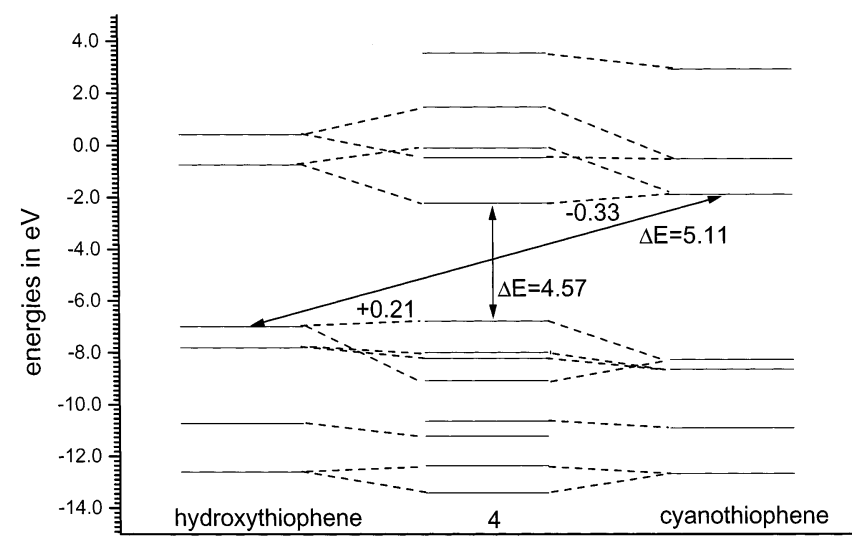

Figure 2. Orbital correlation diagram for $\pi$-orbitals of $\mathbf{4}$ and of hydroxy- and cyanothiophene. The small change of HOMO and LUMO energies shows the effect of large energy difference between IPs and EAs of the interaction fragments.

UNIX versions. ${ }^{80}$ Orbital contours were plotted with the g-openmol program. ${ }^{81}$

\section{Results}

In Figure 1 the energy levels of the $\pi$-orbitals of hydroxyand cyanothiophene are compared with those of thiophene. Hydroxy and cyano groups interact strongly with the lowest all-bonding molecular orbital (MO) of thiophene, forming bonding and antibonding combinations. This interaction leads to a splitting of the lowest energy level into two new levels. In addition the cyano group interacts strongly with the highest lying all antibonding orbital of thiophene, which leads to an additional energy level that lies high in energy. HOMO and LUMO of thiophene are shifted in energy due to substitution, leading to energy differences of $1.27 \mathrm{eV}$ between the HOMOs and of 1.15 $\mathrm{eV}$ between the LUMOs of hydroxy- and cyanothiophene. Thus, alternating hydroxy and cyano substitution should give rise to a polymer with donor-acceptor properties.

In Figure 2 MOs of hydroxy- and cyanothiophene are correlated with those of $\mathbf{4}$. For comparison, the same is done for thiophene and bithiophene in Figure 3. Compared to bithiophene, 4 has three additional energy levels. Two lie in the gap between the four levels arising from HOMO and HOMO-1 and the two levels that stem from the lowest all bonding MO of thiophene. The third extra level is the highest unoccupied molecular orbital. In both systems, the HOMO and LUMO arise from the HOMO and LUMO of thiophene and give rise to the valence and the conduction band upon polymerization. Note that the HOMO-LUMO energy difference 


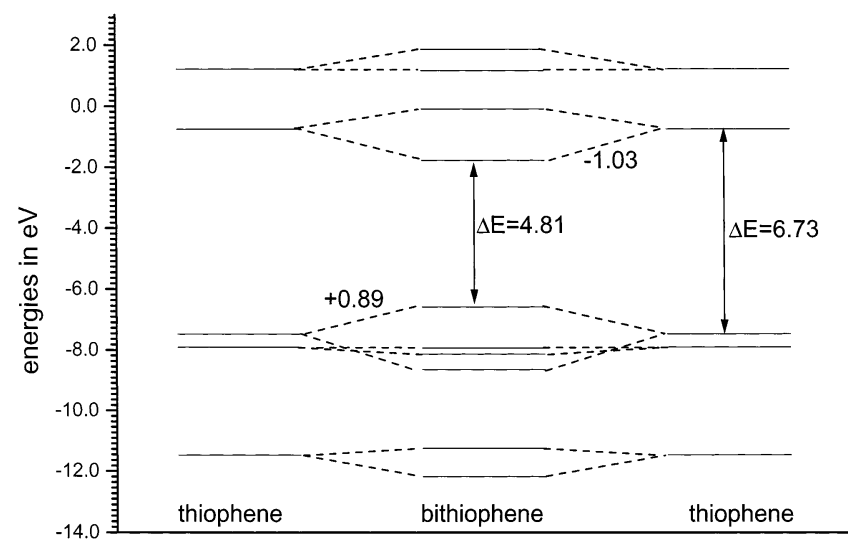

Figure 3. Orbital correlation diagram for thiophene and bithiophene.

TABLE 1: Ionizationpotentials (eV) for Monomers through Tetramers of 1-4

\begin{tabular}{lcccc}
\hline & monomer & dimer & trimer & tetramer \\
\hline $\mathbf{1}$ & 6.59 & 6.04 & 5.86 & 5.78 \\
$\mathbf{2}$ & 7.58 & 7.22 & 7.13 & 7.10 \\
$\mathbf{3}$ & 5.97 & 5.47 & 5.28 & 5.19 \\
$\mathbf{4}$ & 6.79 & 6.37 & 6.23 & 6.17 \\
average 2, 3 & 6.78 & 6.35 & 6.21 & 6.15
\end{tabular}

TABLE 2: Electronaffinities (eV) for Monomers through Tetramers of 1-4

\begin{tabular}{lcccc}
\hline & monomer & dimer & trimer & tetramer \\
\hline $\mathbf{1}$ & 1.78 & 2.46 & 2.71 & 2.82 \\
$\mathbf{2}$ & 3.15 & 3.74 & 4.01 & 4.15 \\
$\mathbf{3}$ & 1.26 & 2.05 & 2.30 & 2.43 \\
$\mathbf{4}$ & 2.22 & 2.88 & 3.15 & 3.29 \\
average 2, 3 & 2.21 & 2.90 & 3.16 & 3.29
\end{tabular}

TABLE 3: Extrapolated Values for IPs, EAs, $E_{\mathrm{g}}, \mathbf{B W}_{\text {val }}$, and $\mathrm{BW}_{\text {cond }}$ for Polymers of 1-4

\begin{tabular}{lccccc}
\hline & IP & EA & $\mathrm{E}_{\mathrm{g}}$ & BW $_{\text {val }}$ & BW $_{\text {cond }}$ \\
\hline $\mathbf{1}$ & 5.53 & 3.20 & 2.33 & 2.35 & 1.99 \\
$\mathbf{2}$ & 7.02 & 4.63 & 2.39 & 2.12 & 1.30 \\
$\mathbf{3}$ & 4.89 & 2.80 & 2.08 & 2.13 & 2.26 \\
$\mathbf{4}$ & 5.98 & 3.74 & 2.22 & 2.13 & 1.63 \\
average 2, 3 & 5.96 & 3.72 & 2.24 & 2.13 & 1.78
\end{tabular}

in thiophene, $6.73 \mathrm{eV}$, is much larger than the energy difference between the HOMO of hydroxythiophene and the LUMO of cyanothiophene, $5.11 \mathrm{eV}$. Nonetheless, the energy gaps in $\mathbf{4}$ and in $\mathbf{1}$ are similar, 4.57 and $4.81 \mathrm{eV}$. Comparison of Figures 2 and 3 shows that the similar energy gaps in $\mathbf{4}$ and in $\mathbf{1}$ arise because the shift in energy levels compared to the fragments is much smaller for $\mathbf{4}(0.54 \mathrm{eV})$ than for $\mathbf{1}(1.92 \mathrm{eV})$.

IPs for monomers through tetramers of $\mathbf{1} \mathbf{- 4}$ are summarized in Table 1. EAs are given in Table 2. Table 3 summarizes extrapolated data for the polymers, and Table 4 lists the $\pi$-charges for the inner rings of the tetramers. All extrapolations were carried with second-degree polynomial fits, since significant deviations from linearity were observed in several cases. Second-degree polynomial fitting leads to near perfect correlation coefficients (0.999 or better). Whenever there was a deviation from linearity, the values changed more rapidly upon polymerization than linear fits would suggest. This is in contrast to experiment where saturation is observed due to disorder. The difference in the energy levels between the cyano- and hydroxysubstituted systems increases upon polymerization. As a result poly-2 has a $2.13 \mathrm{eV}$ larger IP and a $1.83 \mathrm{eV}$ larger EA than poly-3. The band gap of poly-2 is slightly larger than that of polythiophene, and that of poly-3 is slightly smaller. Alternating

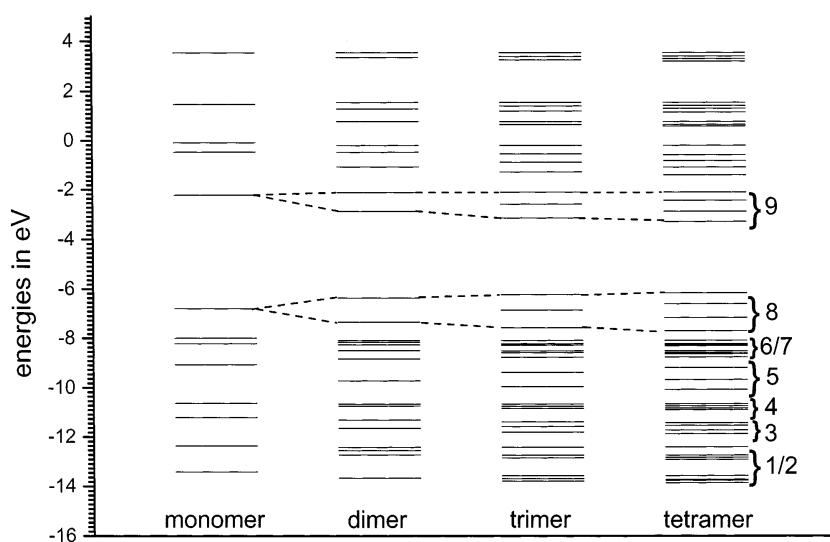

Figure 4. Band development upon chain length increase for oligomers of 4 .

TABLE 4: Charges in the $\pi$-Systems for the Inner Rings of Tetra-2, Tetra-3, Tetra-4, and the Dication of Tetra-4

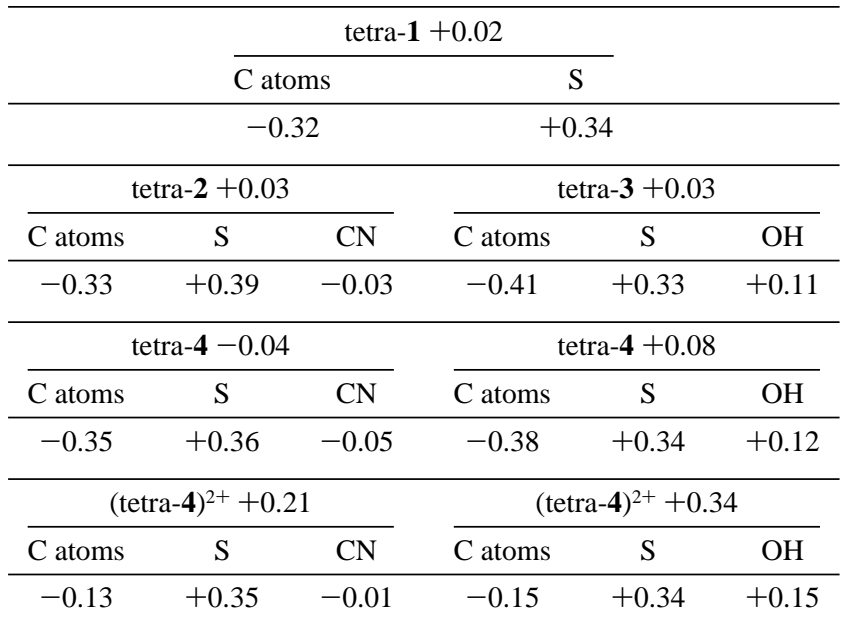

donor-acceptor substitution in poly- $\mathbf{4}$ results in average values for all of the properties at every chain length.

Figure 4 shows the band development with 4 as the repeat unit. With a bicyclic repeat unit, 10 bands arise for polybithiophene (one for each of the energy levels of bithiophene in Figure 3). For the substituted system, 13 bands are formed upon polymerization. With the help of Figures 1 and 2 we can trace the bands back to the $\pi$-orbitals of thiophene. Bands 1 and 2 are similar to those of polybithiophene but extend over the substituents. Bands 3 and 4 are due to substitution and show local charge accumulation (compare orbital contour plots for the trimer of 4 in Figure 5). As a result bands 3 and 4 are flat. Band 5 stems from the HOMO of thiophene and is wide like the valence band 8 that originates from the same orbital. Bands 6 and 7 originate from the HOMO- 1 of thiophene and are flat since the HOMO-1 of thiophene has a node at the $\alpha$-carbon atoms. The conduction band originates from the LUMO of thiophene.

$\mathrm{CN}$ and $\mathrm{OH}$ substitution slightly decrease valence bandwidths compared to polybithiophene. Both systems have nearly the same valence bandwidth. The valence bandwidth of the copolymer is unaffected by alternating donor-acceptor substitution and equals the bandwidths of the homopolymers. The conduction band of poly-2 is narrower than that of polybithiophene; that of poly-3 is wider. The conduction band of the copolymers is narrower than that of polybithiophene and also narrower than the arithmetic mean between those of $\mathbf{2}$ and of 3. To summarize, alternating donor-acceptor substitution in poly-4 leads to average IP, EA, and $E_{\mathrm{g}}$ and tends to decrease 

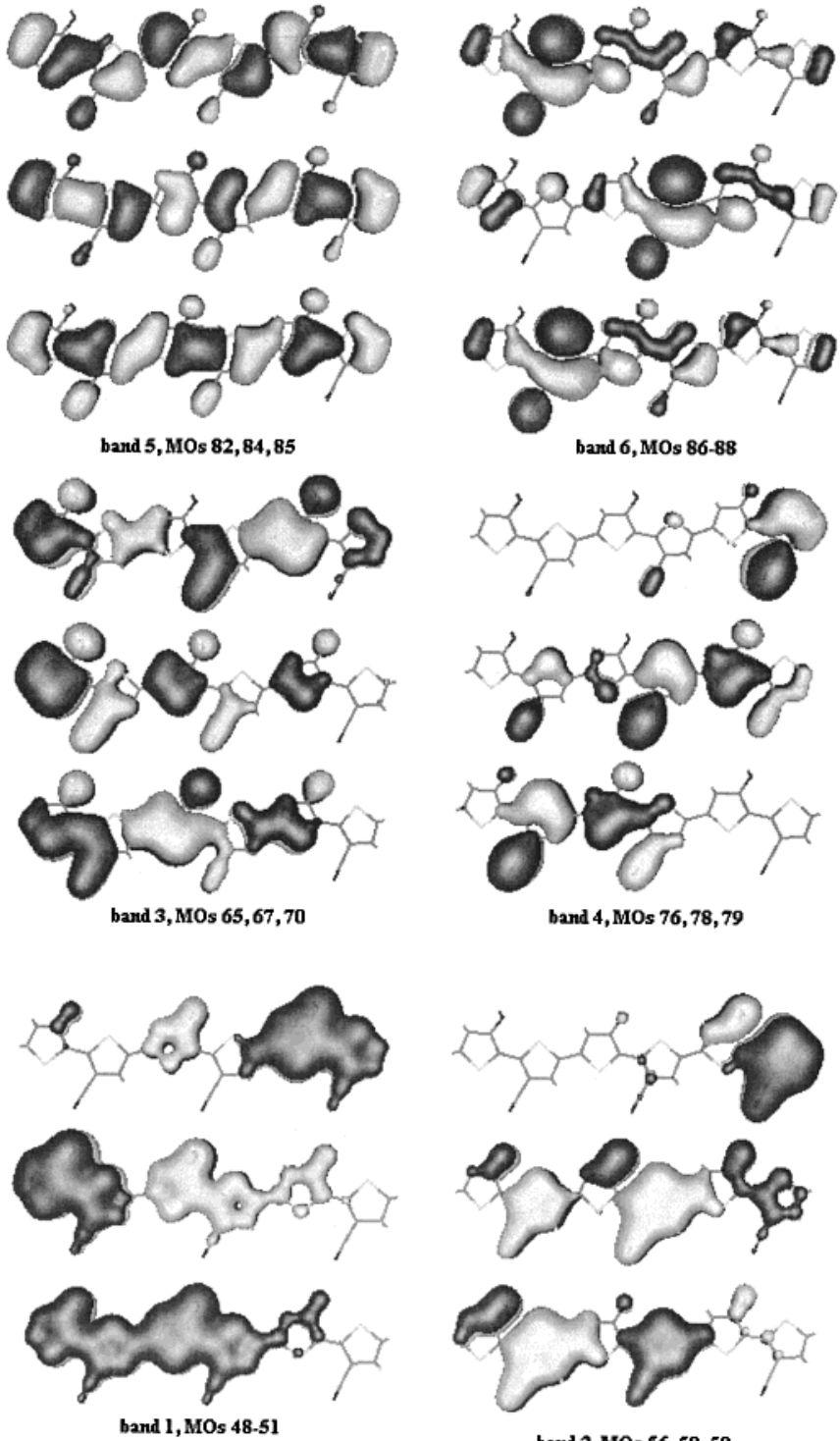

band 2, MOs 56,58,59

Figure 5. Orbital contour plots for the $\pi$-orbitals of the trimer of 4 .

bandwidths compared to the homopolymers. Compared to that of polythiophene, the band gap is reduced by $0.11 \mathrm{eV}$, the valence bandwidth is reduced by $0.22 \mathrm{eV}$, and the conduction bandwidth is reduced by $0.36 \mathrm{eV}$.

In Table $4 \pi$-charges obtained with the NBO method are compiled for the inner rings of the tetramers of $\mathbf{1 - 4}$. In tetra-1, the inner rings are occupied by $5.98 \pi$-electrons. The four carbon atoms possess $4.32 \pi$-electrons and the sulfur lone pair is occupied by $1.66 \pi$-electrons, resulting in $\pi$-charges of +0.02 $\mathrm{e},-0.32 \mathrm{e}$, and $+0.34 \mathrm{e}$, respectively. In tetra-3 the ring (carbon and sulfur atoms) is negatively charged due to $\pi$-donation of 0.11 e from the oxygen lone pair. In tetra- 2 the positive charge of the ring is slightly increased due to the mesomeric effect of the cyano group. It is noteworthy that the carbon atoms do not lose $\pi$-electrons in tetra- 2 but that the sulfur lone pair occupation is reduced to $1.61 \mathrm{e}$. The carbon backbone is still slightly more negative in tetra-2 $(-0.33$ e) than in tetra- $1(-0.32 \mathrm{e})$. When the donor-acceptor system (tetra-4) is formed, some reshuffling of $\pi$-electrons takes place. The carbon atoms of the hydroxythiophene ring lose $0.03 \mathrm{e}$, and the carbon atoms of the cyanothiophene ring gain $0.02 \mathrm{e}$. The sulfur atom of the hydroxythiophene ring becomes slightly more positive, and the sulfur atom of the cyanothiophene ring becomes a little less
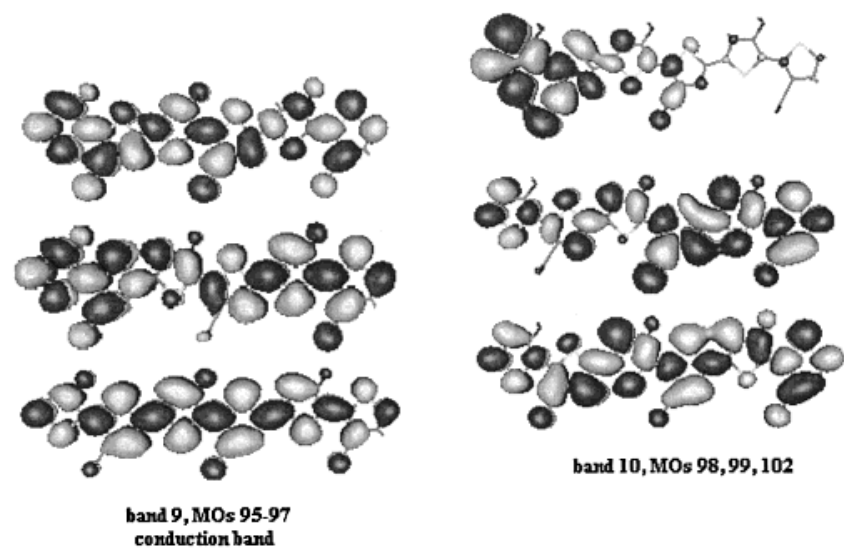

bard 10, MOs 98,99, 102
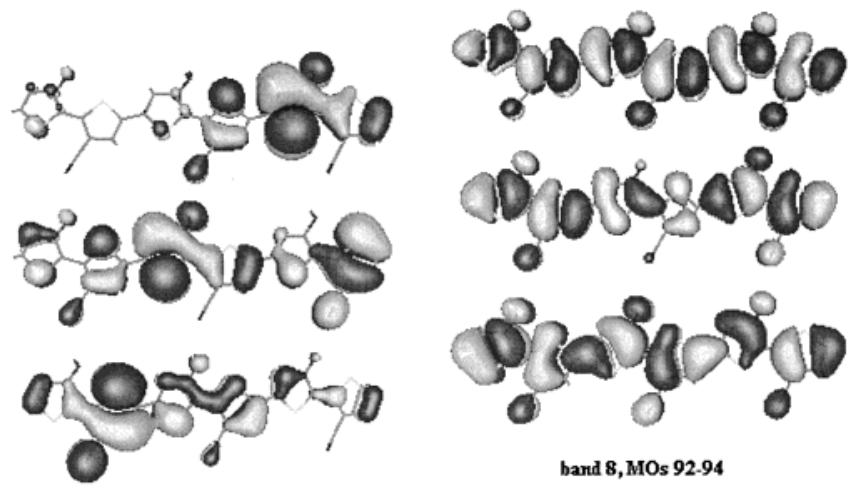

band 7, MOs 89.91

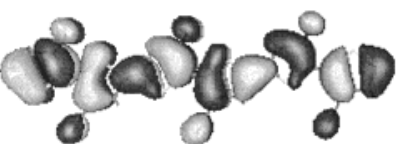

band 8, MOs 92-94 valence band

positive. There does not seem to be a quantitative correlation between charges and shifts in energy levels. This can be attributed to the fact that both $\mathrm{OH}$ and $\mathrm{CN}$ interact with the $\pi$-system through mesomeric rather inductive effects.

\section{Discussion}

The donor-acceptor concept was proposed by Havinga et al. for designing polymers with small band gaps and wide bands. ${ }^{1}$ The idea was justified as follows. If extended regions of donors and acceptors alternate, both valence and conduction bands are curved by space charge effects. A smaller band gap is found when spatial alternation of the level of the bands is taken into account. Havinga et al. realized that a reshuffling of the bands takes place if the extension of the donor and the acceptor regions is decreased. They expected, however, that "at least some broadening of the bands will survive and that the new valence and conduction bands will be broader than in the neutral case, leading to a smaller band gap." The idea is illustrated in Figure 6 using calculated values for poly-1, -2, $\mathbf{- 3}$, and $\mathbf{- 4}$. Data for poly-2 and for poly-3 show that the valence and conduction bands are strongly shifted in energy due to substitution with $\mathrm{OH}$ and $\mathrm{CN}$ groups. The wide bands indicated with donor-acceptor show a hypothetical situation in which 


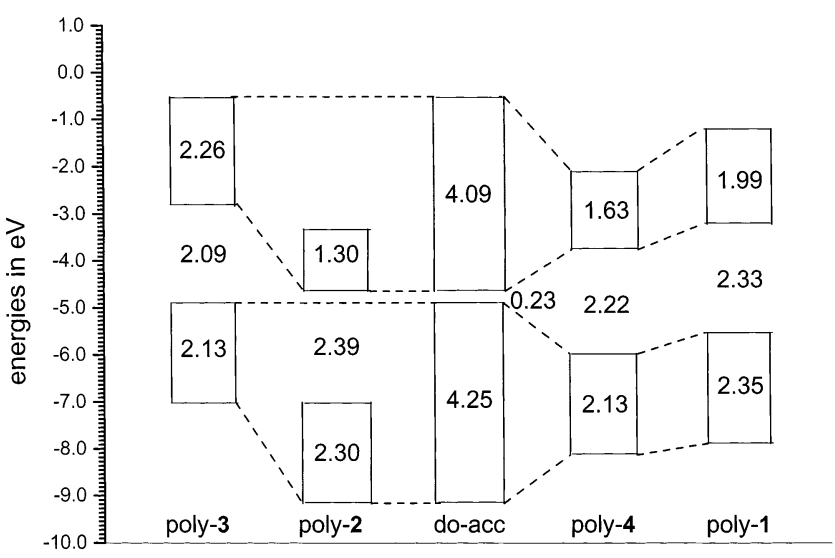

Figure 6. Illustration of the donor-acceptor concept using calculated data for poly-2, poly-3, and poly-4. Donor-acceptor substitution should tend to increase bandwidths and decrease the band gap, as shown for donor-acceptor as a hypothetical extreme case. In contrast, bandwidths are decreased and the band gap is average between those of the homopolymers.

the valence band of the copolymer spans the whole range of valence bands of donor and acceptor and the conduction band spans the whole range of the conduction bands of donor and acceptor. This indeed would lead to a small band gap, $0.23 \mathrm{eV}$, and large bandwidths, 4.25 and $4.09 \mathrm{eV}$. If the donor acceptor concept worked, the band edges of poly- $\mathbf{4}$ should lie somewhere between those of the homopolymers and those of the hypothetical donor-acceptor polymer. The last two entries in Figure 4 show data for poly-4 and for poly-1. The comparison reveals that Havinga et al.'s prediction is not borne out for poly-4. No band broadening is observed for poly-4 compared to either homopolymer or polythiophene. In contrast, both valence and conduction bands are narrower than those of polythiophene. The band gap is slightly smaller than that of polythiophene but precisely average between those of the homopolymers. These findings are in agreement with experimental findings that oxidation potentials and $\pi-\pi^{*}$ transitions in the methoxy analogue of $\mathbf{4}$ and polybithiophene occur at similar energies. ${ }^{5,19}$

Why are the bands not getting wider as suggested by the donor-acceptor concept in poly-4 and why is the band gap not reduced substantially? The reason becomes apparent if the system is built up from the repeat units rather than starting from polymers and gradually reducing the extent of the donor and acceptor regions. Upon formation of $\mathbf{4}$ the HOMO increases by $0.21 \mathrm{eV}$ and the LUMO decreases by $0.33 \mathrm{eV}$ (compare Figure 2), resulting in a HOMO-LUMO gap reduction of 0.54 $\mathrm{eV}$. When two thiophene rings interact (Figure 3), the HOMO energy increases by $0.89 \mathrm{eV}$, the LUMO energy decreases by $1.03 \mathrm{eV}$, and the energy gap decreases by $1.92 \mathrm{eV}$. Thus, when the $\mathbf{4}$ is formed, alternating donor-acceptor substitution leads to $1.38 \mathrm{eV}$ less reduction in the energy gap compared to the unsubstituted bithiophene. This can be rationalized with perturbation theory. The energy change due to a perturbation is directly proportional to the square of the Fock matrix element between the interaction orbitals and indirectly proportional to the energy difference $\left(\epsilon_{\mathrm{i}}-\epsilon_{\mathrm{j}}\right)$ between the interacting orbitals:

$$
\Delta E \sim\left\langle F_{i j}\right\rangle^{2} / \epsilon_{i}-\epsilon_{j}
$$

$F_{i j}$, the Fock matrix element between the interacting orbitals is roughly proportional to overlap. Therefore, assuming constant overlap, the interaction energy decreases as the energy difference increases. Figures 2 and 3 look like textbook examples of orbital energy plots for interaction of energy levels with very different and with identical energies. ${ }^{82}$ The larger the electronegativity difference, the smaller the change in energy levels. Generalizing the two-level case to a many-level case as in conducting polymers, the result of donor-acceptor interaction is narrowing of bands and less decrease of energy gap upon polymerization. Thus, perturbation theory rationalizes experimental and theoretical results but disagrees with the donor-acceptor concept. It seems that band gap reduction can be accomplished with the donor-acceptor concept only at the expense of bandwidth.

A second but much smaller effect contributes also. Independently of the donor-acceptor substitution, HOMO and LUMO levels shift to a different extent upon polymerization in poly-2 and in poly-3 compared to polythiophene. The energy gap decrease between monomers and polymers is $2.48 \mathrm{eV}$ for poly1, $2.04 \mathrm{eV}$ for poly-2, $2.62 \mathrm{eV}$ for poly-3, and $2.33 \mathrm{eV}$ for poly4. The decrease for poly-4 is the average between those for poly-2 and poly-3 and $0.15 \mathrm{eV}$ smaller than in poly-1.

Inspection of the population of the $\mathrm{p}_{z}$-orbitals of the $\alpha$-carbon atoms shows that cyano substitution decreases the $\pi$-electron density at that position; hydroxy substitution increases it compared to the unsubstituted parent. This correlates with a decrease in overlap in the valence band of poly-2 and a decrease in shift of the HOMO energy. In poly-3 an increase in overlap in the valence band and a larger shift of the HOMO energy results. We can thus assign about $0.15 \mathrm{eV}$ in reduced band gap decrease in poly- 4 to a similar change in electron density at the $\alpha$-carbon atoms. This has nothing to do with donor-acceptor substitution.

To summarize, polythiophene has a $4.40 \mathrm{eV}$ smaller energy gap than thiophene. Poly-4 has an energy gap that is $2.87 \mathrm{eV}$ smaller than the energy difference between the HOMO of hydroxythiophene and the LUMO of cyanothiophene. Most of this difference is due to a reduced shift in energy when HOMOs and LUMOs of very different energies interact. This fact was overlooked in the design of the donor-acceptor concept, which implies that band development is not substantially altered by connecting donors and acceptors with very different energy levels.

In their original publication Havinga et al. stressed the importance of charge transfer. In poly- $\mathbf{4}$ such charge transfer is calculated but it is not accompanied by a decrease in band gap. Theoretical analysis by Brocks showed that there is no charge transfer in poly(aminosquaraine), where a substantial decrease in bang gap was accomplished. In contrast, hybridization between the HOMO/LUMO states of the fragments was shown to play the decisive role, leading to the small band gap. One has to be careful, therefore, not to attribute changes in energy levels solely to changes in charges. Charges were an important factor, if the shift in energy levels was due to inductive effects. However, energy levels can also be shifted through mesomeric effects. For instance, the cyano group lowers the HOMO energy in tetra- 2 by $1.13 \mathrm{eV}$, although the $\pi$-charge (as well as and total charge) on $\mathrm{CN}$ is only $-0.03 \mathrm{e}$. The increase in $\mathrm{HOMO}$ energy due to hydroxy substitution is smaller, $+0.59 \mathrm{eV}$, although the $\pi$-charge of the hydroxy group is +0.11 e. Note that the total charge $(\sigma$ and $\pi$ ) of the hydroxy group is -0.22 e. There is no quantitative correlation between charge shift, $\sigma$ or $\pi$, and shift in energy levels. It is not surprising, therefore, that the HOMO of tetra-4 is lower in energy than that of tetra-1 despite the mean positive $\pi$-charge $(+0.02$ e) over the hydroxy and cyano substituted rings.

Demanze et al. observed peculiar electrochemical behavior of the methoxy analogue of poly-4 upon p-doping and interpreted their findings in terms of a quantum well structure..$^{5,19}$ The $\pi$-charges of the cyano- and the hydroxy-substituted 
thiophene rings in neutral tetra- $\mathbf{4}$ differ by $0.12 \mathrm{e}$. This charge difference, however, is not a measure of a possible quantum well character in individual bands, since the $\pi$-occupations consist of the sum of the $\pi$-electrons in all $\pi$-bands. Orbital contour plots in Figure 5 show that valence and conduction bands are delocalized over the whole molecule. No visible localization of electron density takes place in valence and conduction bands. In contrast, lower energy levels appear to be localized. For instance band 1 has larger contributions from hydroxy rings than from cyano rings. Bands 2 and 4 are essentially localized at the cyano rings. That this localization occurs only in the low-lying levels is due to the fact that hydroxy and cyano groups interact most strongly with the lowest MO of thiophene (Figure 1). Since the valence band is delocalized and since it is this band that loses its electrons upon ionization, no additional charge localization upon p-doping is predicted at this level of theory. Actual calculation shows that in the dication of tetra-4, the innermost cyano ring carries a $\pi$-charge of +0.21 $\mathrm{e}$, the innermost hydroxy ring has a $\pi$-charge of $+0.34 \mathrm{e}$. There is thus a similar charge separation, $0.13 \mathrm{e}$, in the dication as in the neutral species $(0.12 \mathrm{e})$.

\section{Conclusions}

DFT calculations on poly-4 agree with experimental findings on poly(3-methoxy-3'-cyanobithiophene) regarding band gap and IP. In contrast to expectations based on the donor-acceptor concept, alternating donor-acceptor substitution in poly-4 does not decrease the band gap compared with the homopolymers and does not increase valence and conduction bandwidths. Electronic properties of the copolymer are close to the arithmetic mean between those of the homopolymers. Both neutral poly-4 and the dication of poly-4 exhibit uneven charge distribution, with hydroxy rings being more positively charged than cyano rings, but the charge separation is not increased in the dication compared to the neutral polymer.

The reason for the lack of band broadening in poly-4 is that the strength of interaction between fragments is inversely proportional to their energy difference, as predicted by perturbation theory. The result is less decrease in band gap and less increase in bandwidths upon polymerization compared to homopolymers. We expect therefore the donor-acceptor concept to lead to an inverse proportionality between band gap reduction and bandwidths. This has negative consequences for conductivity.

Poly-4 represents a donor-acceptor system in which overlap between fragments at the $\alpha$-carbon atoms is large and in which charge transfer occurs from the donor to the acceptor. The difference between IPs and EAs in the fragments is due to mesomeric effects. It remains to be established whether systems in which inductive effects are dominant show different behavior. Additional effects are conceivable when a symmetry mismatch between the frontier orbitals occurs, and when building blocks with quinoid structures are used. In the paper following this one, a system is investigated in which the coefficients at the $\alpha$-carbon atoms are small in the conduction band. Work on various kinds of donor-acceptor systems is in progress.

Acknowledgment. I thank Bilkent University and TÜBITAK (TBAG 1887) for providing funding for computational resources.

\section{References and Notes}

(1) Havinga, E. E.; ten Hoeve, W.; Wynberg, H. Synth. Met. 1993, $55-57,299-306$
(2) Tol, A. J. W. J. Chem. Phys. 1994, 100, 8463-8470.

(3) Tamao, K.; Yamaguchi, S.; Yoshihiko, I. J. Chem. Soc., Chem. Commun. 1994, 229-230.

(4) Kitamura, C.; Tanaka, S.; Yamashita, Y. J. Chem. Soc., Chem. Commun. 1994, 1585-1586. 910.

(5) Demanze, F.; Yassar, A.; Garnier, F. Adv. Mater. 1995, 7, 907-

(6) Brocks, G. J. Chem. Phys. 1995, 102, 2522-2532.

(7) Karikomi, M.; Kitamura, C.; Tanaka, S.; Yamashita, Y. J. Am Chem. Soc. 1995, 117, 6791-6792.

(8) Ho, H. A.; Brisset, H.; Frère, P.; Roncali, J. J. Chem. Soc., Chem. Commun. 1995, 2309-2310.

(9) Kanbara, T.; Miyazaki, Y.; Yamamoto, T. J. Polym. Sci. A 1995 33, 999-1003.

(10) Cornil, J.; dos Santos, D. A.; Beljonne, D.; Brédas, J. L. J. Phys. Chem. 1995, 99, 5604-5611.

(11) Brocks, G.; Tol, A. J. Phys. Chem. 1996, 100, 1838-1846.

(12) Bakhshi, A. K.; Yamaguchi, Y.; Ago, H.; Yamabe, T. Synth. Met 1996, 79, 115-120.

(13) Yamamoto, T.; Zhou, Z.-h.; Kanbara, T.; Shimura, M.; Kizu, K.; Maruyama, T.; Nakamura, Y.; Fukuda, T.; Lee, B.-L.; Ooba, N.; Tomaru, S.; Kurihara, T.; Kaino, T.; Kubota, K.; Sasaki, S. J. Am. Chem. Soc. 1996, 118, 10389-10399.

(14) Suzuki, Y. Y.; Beljonne, D.; Brédas, J. L. J. Chem. Phys. 1996 $104,7270-7283$

(15) van Mullekom, H. A. M.; Vekemans, J. A. J. M.; Meijer, E. W. Chem. Commun. 1996, 2163.

(16) Tamao, K.; Ohno, S.; Yamaguchi, S. J. Chem. Soc., Chem. Commun. 1996, 1873-1874.

(17) Nobutoki, H.; Koezuka, H. J. Phys. Chem. 1996, 100, 6451-6455. 64

(19) Demanze, F.; Yassar, A.; Garnier, F. Macromolecules 1996, 29, 4267-4273.

(20) Bublitz, G. U.; Ortiz, R.; Runser, C.; Fort, A.; Barzoukas, M.; Marder, S. R.; Boxer, S. G. J. Am. Chem. Soc. 1997, 119, 2311-2312.

(21) Zhang, T. Q.; Tour, J. M. J. Am. Chem. Soc. 1997, 119, 50655066 .

(22) Duan, X.; Kawai, R.; Yeates, A. T.; Dudis, D. S. Synth. Met. 1997, $85,1153-1154$

(23) Tanaka, S.; Yamashita, Y. Synth. Met. 1997, 84, 229-230.

(24) Alemán, C.; Domingo, V. M.; Fajarí, L.; Juliá, L.; Karpfen, A. J. Org. Chem. 1998, 63, 1041-1048.

(25) Zhang, Q. T.; Tour, J. M. J. Am. Chem. Soc. 1998, 120, 53555362 .

(26) Huang, H.; Pickup, P. G. Chem. Mater. 1998, 10, 2212-2216.

(27) Kertesz, M.; Choi, C. H.; Sun, G. Polym. Prepr. (Am. Chem. Soc., DIv. Polym. Chem.) 1998, 39, 76.

(28) Hucke, A.; Cava, M. P. J. Org. Chem. 1998, 63, 7413-7417.

(29) Yu, W.-L.; Meng, H.; Pei, J.; Lai, Y.-H.; Chua, S.-J.; Huang, W. J. Chem. Soc., Chem. Commun. 1998, 1957-1958.

(30) Akoudad, S.; Roncali, J. J. Chem. Soc., Chem. Commun. 1998, 2081-2082.

(31) van Mullekom, H. A. M.; Vekemans, J. A. J. M.; Meijer, E. W. Polym. Prepr. (Am. Chem. Soc., DIv. Polym. Chem.) 1998, 39, 10021003.

(32) Bakhshi, A. L.; Rattan, P. J. Chem. Soc., Faraday Trans. 1998, 94, 2823-2826.

(33) DuBois, C. J.; Irvin, D. J.; Reynolds, J. R. Polym. Prepr. (Am. Chem. Soc., DIv. Polym. Chem.) 1998, 39, 1214.

(34) Yu, W.-L.; Meng, H.; Pei, J.; Huang, W. J. Am. Chem. Soc. 1998, 120, 11808-11809.

(35) Meng, H.; Chen, Z.-K.; Huang, W. J. Phys. Chem. B 1999, 103 , 6429-6433.

(36) Chenthamarakshan, C. R.; Eldo, J.; Ajayaghosh, A. Macromolecules 1999, 32, 251-257.

(37) Breitung, E. M.; Shu, C.-F.; McMahon, R. J. J. Am. Chem. Soc. 2000, 122, 1154-1160.

(38) Salzner, U. Synth. Met. 2001, 119, 215-216.

(39) Belletête, M.; Morin, J.-F.; Beaupré, S.; Ranger, M.; Leclerc, M.; Durocher, G. Macromolecules 2001, 34, 228-2297.

(40) Jenekhe, S. A.; Lu, L.; Alam, M. M. Macromolecules 2001, 34, 7315-7324

(41) van Mullekom, H. A. M.; Vekemans, J. A. J. M.; Havinga, E. E.; Meijer, E. W. Mater. Sci. Eng. 2001, R32, 1-40.

(42) Naitoh, S.; Sanui, K.; Ogata, N. J. Chem. Soc., Chem. Commun. 1986, $1348-1350$.

(43) Bakhshi, A. K.; Ladik, J.; Seel, M. Phys. Rev. B 1987, 35, 704712.

(44) Garnier, F. Angew. Chem., Int. Ed. Engl. 1989, 28, 513-517.

(45) Ferraris, J. P.; Hanlon, T. R. Polymer 1989, 30, 1319-1327. 
(46) Zhou, Z.-H.; Maruyama, T.; Kanbara, T.; Ikeda, T.; Ichimura, K.; Yamamoto, T.; Tokuda, K. J. Chem. Soc., Chem. Commun. 1991, 12101212.

(47) Burn, P. L.; Holmes, A. B.; Kraft, A.; Bradley, D. D. C.; Brown,

A. R.; Friend, R. H.; Gymer, R. W. Nature 1992, 356, 47-49.

(48) Meyers, F.; Heeger, A. J.; Brédas, J. L. J. Chem. Phys. 1992, 97, 2750-2758.

(49) Yamamoto, T.; Shimura, M.; Osakada, K.; Kubota, K. Chem. Lett. 1992, 1003-1004

(50) Musmanni, S.; Ferraris, J. P. J. Chem. Soc., Chem. Commun. 1993, $172-174$.

(51) Greenham, N. C.; Moratti, S. C.; Bradley, D. D. C.; Friend, R. H.; Holmes, A. B. Nature 1993, 365, 628-630.

(52) Tanaka, S.; Yamashita, Y. Synth. Met. 1993, 55-57, 1251-1254.

(53) Meyers, F.; Heeger, A. J.; Brédas, J. L. Synth. Met. 1993, 55-57, 4308-4313.

(54) Naarmann, C. H.; Theophilou, N. Synth. Met. 1987, 22, 1.

(55) Kaiser, A. B. Adv. Mater. 2001, 13, 927-941.

(56) Roncali, J.; Yassar, Y.; Garnier, F. J. Chem. Soc., Chem. Commun. 1988, $581-582$

(57) Salzner, U. J. Mol. Model. 2000, 6, 195-204

(58) Becke, A. D. Phys. Rev. A 1988, 38, 3098-3100.

(59) Perdew, J. P. Phys. Rev. B 1986, 33, 8822-8824.

(60) Salzner, U.; Lagowski, J. B.; Pickup, P. G.; Poirier, R. A. J. Phys. Chem. 1998, 102, 2572-2578.

(61) Stevens, W.; Basch, H.; Krauss, J. J. Chem. Phys. 1984, 81, 6026.

(62) Baerends, E. J.; Gritsenko, O. V. J. Phys. Chem. A 1997, 101, 5383-5403.

(63) Casida, M. E.; Jamorski, C.; Casida, K. C.; Salahub, D. R. J. Chem. Phys. 1998, 108, 4439.

(64) Chan, G. E.-L.; Tozer, D. J.; Handy, N. C. J. Chem. Phys. 1997, 107, 1536.

(65) Fritsche, L. Physica B 1991, 172, 7-17. 863.

(66) Galbraith, J. M.; Schaefer, H. F. I. J. Chem. Phys. 1996, 105, 862-

(67) Godby, R. W.; Schlüter, M.; Sham, L. J. Phys. Rev. B 1988, 37, 10159-10175.
(68) Levy, M. Phys. Rev. A 1995, 52, 50-52.

(69) Malkin, V. G.; Malkina, O. L.; Casida, M. E.; Salahub, D. R. J. Am. Chem. Soc. 1994, 116, 5898-5908.

(70) Perdew, J. P.; Levy, M. Phys. Rev. Lett. 1983, 51, 1884.

(71) Rösch, N.; Trickey, S. B. J. Chem. Phys. 1997, 106, 8940-8941.

(72) Salzner, U.; Lagowski, J. B.; Pickup, P. G.; Poirier, R. A. J. Comput. Chem. 1997, 18, 1943-1953.

(73) Stowasser, R.; Hoffmann, R. J. Am. Chem. Soc. 1999, 121, 34143420 .

(74) Williams, A. R.; von Barth, U. In Theory of the Inhomogeneous Electron Gas; Lundqvist, S., March, N. H., Eds.; Plenum Press: London, 1983.

(75) Brunck, T. K.; Weinhold, F. J. Am. Chem. Soc. 1978, 100, 1700

(76) Foster, J. P.; Weinhold, F. J. Am. Chem. Soc. 1980, 102, 7211.

(77) Reed, E. A.; Weinstock, R. B.; Weinhold, F. J. Chem. Phys. 1985, 83,735 .

(78) Reed, A. E.; Weinhold, F. J. Chem. Phys. 1985, 83, 1736.

(79) Reed, E. A.; Curtiss, L. A.; Weinhold, F. Chem. Rev. 1988, 88, 899.

(80) Frisch, M. J.; Trucks, G. W.; Schlegel, H. B.; Scuseria, G. E.; Robb, M. A.; Cheeseman, J. R.; Zakrzewski, V. G.; Montgomery, J., J. A.; Stratmann, R. E.; Burant, J. C.; Dapprich, S.; Millam, J. M.; Daniels, A. D.; Kudin, K. N.; Strain, M. C.; Farkas, O.; Tomasi, J.; Barone, V.; Cossi, M.; Cammi, R.; Mennucci, B.; Pomelli, C.; Adamo, C.; Clifford, S.; Ochterski, J.; Petersson, G. A.; Ayala, P. Y.; Cui, Q.; Morokuma, K.; Malick, D. K.; Rabuck, A. D.; Raghavachari, K.; Foresman, J. B.; Cioslowski, J.; Ortiz, J. V.; Stefanov, B. B.; Liu, G.; Liashenko, A.; Piskorz, P.; Komaromi, I.; Gomperts, R.; Martin, R. L.; Fox, D. J.; Keith, T.; Al-Laham, M. A. Peng, C. Y.; Nanayakkara, A.; Gonzalez, C.; Challacombe, M.; Gill, P. M. W.; Johnson, B.; Chen, W.; Wong, M. W.; Andres, J. L.; Gonzalez, C.; Head-Gordon, M.; Replogle, E. S.; Pople, J. A. Gaussian 98, revision A.3; Gaussian, Inc.: Pittsburgh, PA, 1998.

(81) Laaksonen, L. http://laaksonen.csc.fi/gopenmol/, 1999.

(82) Atkins, P. W.; Friedman, R. S. Molecular Quantum Mechanics; Oxford University Press: Oxford, U.K., 2000. 